\title{
Der Einfluss von Fritz Oser auf vier Themen der Unterrichts- und Schulforschung: eine dankbare Rückschau und ein Blick in die Zukunft
}

\author{
Cornelia Gräsel • Knut Neumann · Hans Gruber • Martin Rothgangel • \\ Manfred Prenzel
}

Angenommen: 1. Februar 2021 / Online publiziert: 16. Februar 2021

(C) Der/die Autor(en) 2021

\section{Einleitung (Cornelia Gräsel)}

Am 5. September 2020 ist Fritz Oser, der große Schweizer Wissenschaftler, Pädagoge und Psychologe, verstorben. Mit seinem Tod haben viele Unterrichtsforscher*innen einen wichtigen Ideengeber und Begleiter verloren. Denn Fritz Oser hat mit seiner lebhaften Präsenz auf Tagungen und kollegialen Treffen nicht nur seine Schüler*innen beeinflusst, sondern einen großen Kreis von Wissenschaftler*innen. Begegnungen mit Fritz Oser waren für viele von uns inspirierend oder gar prägend, weil er als Persönlichkeit wirkte: mit scheinbar endloser Energie, mit einem Schatz an zu teilenden Erfahrungen, mit hintergründigem Humor und mit einem pädago-

\footnotetext{
C. Gräsel $(\bowtie)$

Institut für Bildungsforschung, Bergische Universität Wuppertal, Gaußstr. 20, 42117 Wuppertal,

Deutschland

E-Mail: graesel@uni-wuppertal.de

K. Neumann

Didaktik der Physik, IPN-Leibniz-Institut für die Pädagogik der Naturwissenschaften und Mathematik, Olshausenstr. 62, 24118 Kiel, Deutschland

E-Mail: neumann@leibniz-ipn.de

H. Gruber

Institut für Erziehungswissenschaft, Universität Regensburg, Universitätsstraße 31, 93053 Regensburg, Deutschland

E-Mail: Hans.Gruber@ur.de

M. Rothgangel

Evangelisch-Theologische Fakultät, Institut für Religionspädagogik, Universität Wien, Schenkenstr. 8-10, 1010 Wien, Österreich

E-Mail: martin.rothgangel@univie.ac.at

M. Prenzel

Zentrum für LehrerInnenbildung, Universität Wien, Porzellangasse 4, 1090 Wien, Österreich

E-Mail: manfred.prenzel@univie.ac.at
} 
gischen Denken, das nicht nur analytische Klarheit, sondern auch Richtung und Orientierung offerierte.

Noch viel stärker als in persönlichen Begegnungen hat Fritz Oser durch sein herausragendes und vielfältiges akademisches Werk gewirkt, das in diesem Beitrag gewürdigt werden soll. Das Oeuvre von Fritz Oser ist dabei so umfassend, dass ein Überblick und eine Einordnung nicht von einer einzelnen Person geleistet werden kann. Vier Themenfelder werden daher im Folgenden von vier Experten daraufhin analysiert, wie Fritz Oser sie geprägt hat und auch weiter prägen wird. (1) Knut Neumann wird als Mitherausgeber dieser Zeitschrift mit dem zentralen Thema der „Unterrichtswissenschaft“ beginnen, nämlich der Forschung zur Unterrichtsqualität. (2) Das Lernen aus Fehlern und ein produktiver Umgang mit Fehlern ist nicht nur im Unterricht eine wichtige Form der Erkenntnis - Hans Gruber gibt aus der Perspektive eines wissenschaftlichen Freundes von Fritz Oser dazu einen Überblick. (3) Martin Rothgangel kann als Religionspädagoge zeigen, wie stark Fritz Osers Arbeiten Forschungen zu moralischer Erziehung und $\mathrm{zu}$ just communities beeinflusst haben. (4) In den letzten Jahren hat sich Fritz Oser mit großem Engagement der Weiterentwicklung der Lehrpersonenbildung gewidmet; dieses Thema wird von Manfred Prenzel betrachtet, der Fritz Oser durch das Streben nach wissenschaftlich begründeten Reformen verbunden war.

Insgesamt verfolgen wir mit diesem Beitrag nicht nur das Anliegen, das Werk von Fritz Oser in seiner Innovativität und Größe in Erinnerung zu rufen. Wir hegen auch die Hoffnung, dass diese Zusammenschau Kolleg*innen dazu anregen kann, seine Ideen aufzugreifen und durch empirische Forschung weiterzuentwickeln.

\section{Der Gral der Unterrichtsforschung: Fritz Osers Choreographien unterrichtlichen Lernens (Knut Neumann)}

Das große Ziel der Unterrichtsforschung ist, Merkmale guten Unterrichts zu identifizieren, und dabei bestenfalls die individuellen Voraussetzungen der Schüler*innen, die Eigenschaften der Lehrkraft sowie die schulischen Rahmenbedingungen zu berücksichtigen. Zwischen diesem Anspruch und der Realität klaffte lange Jahre eine große Lücke. So wurde (guter) Unterricht bis zu den 1990er-Jahren im Wesentlichen als Konvolut verschiedener Merkmale gesehen - manche mit nachgewiesener Wirkung auf Lernen, wie z.B. time-on-task, andere wie z.B. Gruppenarbeit eher einem Wunschdenken entspringend. Dass dies heute weniger der Fall ist, ist ein wesentliches Verdienst Fritz Osers. Er hat 1) mit der Unterscheidung von Oberflächenund Tiefenstruktur die Erweiterung des Begriffs der Unterrichtsqualität um eine Dimension jenseits oberflächlicher Merkmale begründet, 2) durch die Beschreibung von zwölf prototypischen Abfolgen von Handlungsschritten zur (erfolgreichen) Erarbeitung eines breiten Spektrums von Lernzielen einen wesentlichen Beitrag zur empirischen Unterrichtsforschung geleistet, und damit in der Folge 3) wesentliche Erkenntnisse für die empirisch fundierte Verbesserung der Lehrer*innenaus- und Weiterbildung und in der Folge auch des Unterrichts beigetragen. 


\subsection{Oberflächen- und Tiefenstruktur - Erweiterung des Begriffs der Unterrichtsqualität}

Osers erster wesentlicher Beitrag zur Unterrichtsforschung ist die Unterscheidung zweier Ebenen von Unterricht: der Oberflächen- und der Tiefenstruktur (Oser und Patry 1990, 1994; Oser und Baeriswyl 2001). Die Sicht- oder auch Oberflächenstruktur von Unterricht umfasst die direkt beobachtbaren, frei zu gestaltenden Elemente des Unterrichts wie z. B. Unterrichtsform, Methoden oder Medien. Die Basis- oder auch Tiefenstruktur besteht aus einer nicht direkt beobachtbaren Folge von Lernschritten. Idealerweise sind diese kongruent zu einer durch Lernziel und lernpsychologische Gesetzmäßigkeiten festgelegten Abfolge. Fritz Oser selbst führte die einprägsame Analogie zum Tanzen an: Während ein*e Tänzer*in an die durch die Choreographie festgelegten Tanzschritte gebunden ist, ist er*sie frei in seiner Interpretation des Tanzes. Wie ein*e Tänzer*in muss eine Lehrkraft für guten Unterricht einer durch das Lernziel festgelegten (schematischen) Abfolge von Handlungsschritten folgen, wie sie diese methodisch umsetzt, oder welche Medien sie dabei einsetzt, bleibt aber ihr überlassen; hier können verschiedene Varianten zum Erfolg führen. Damit löste Fritz Oser die Spannung auf zwischen der im anglo-amerikanischen Raum prominenten Vision eines schematisch geplanten und umgesetzten Unterrichts und der im deutschsprachigen Raum verbreiteten Vorstellung, dass guter Unterricht viele Varianten hat. Das feste Schema ist Merkmal der Tiefenstruktur, die Varianten finden sich in der Oberflächenstruktur. Diese Trennung von Oberflächen- und Tiefenstruktur hat die Unterrichtsforschung nachhaltig verändert. Historisch lag der Fokus der Unterrichtsforschung vielfach auf der Wirksamkeit von Merkmalen der Oberflächenstruktur. Insbesondere in der deutschsprachigen Didaktik wurde den Methoden eine zentrale Bedeutung beigemessen (Frey et al. 1988). Dieser Fokus verschob sich in Folge der wegweisenden Arbeit Fritz Osers auf die Erforschung von Merkmalen der Tiefenstruktur, darunter kognitive Aktivierung, konstruktive Unterstützung und effektive Klassenführung (z. B. Drollinger-Vetter 2011; Gebauer 2016; Prenzel et al. 2002; für einen Überblick siehe Praetorius et al. 2020).

\subsection{Basismodelle des Unterrichts - Beitrag zur empirischen Unterrichtsforschung}

Der zweite wesentliche Beitrag Fritz Osers zur Unterrichtsforschung liegt in der Beschreibung von zwölf Modellen der Tiefenstruktur guten Unterrichts - den so genannten Basismodellen (Oser und Patry 1990, 1994; Oser und Baeriswyl 2001). Noch vor der Beschreibung von Mustern guten Unterrichts im Rahmen der TIMSS Videostudie destillierte Fritz Oser mit seiner Arbeitsgruppe aus Beobachtungen von über einhundert Unterrichtsstunden zu jedem der zwölf Basismodelle eine idealtypische Abfolge von Handlungsschritten heraus (z. B. Wissensaufbau, Problemlösen). Für das Basismodell Wissensaufbau sind dies: 1) Direkte oder indirekte Bewusstmachung des Vorwissens, 2) Vorstellen und Durcharbeiten eines Prototyps (dessen Erarbeitung mehr als das bestehende Wissen erfordert), 3) Präsentation bzw. Repräsentation einer oder mehrerer neuer Wissenselemente (die zur Erarbeitung des Phänomens notwendig sind), 4) Eingliederung der neuen Elemente in die bestehende 
Wissensstruktur und 5) Schaffung einer optimalen Koordination innerhalb der neuen Wissensstruktur. Mit der Beschreibung der zwölf Basismodelle, die jeweils individuelle Lernziele adressieren sowie eine entsprechende Sequenz von Handlungsschritten im Unterricht und damit eine idealtypische Abfolge von Lernschritten definieren, legte Fritz Oser als erster eine umfassende Beschreibung prototypischer Muster guten Unterrichts vor. Diese decken sowohl fachspezifische als auch fachübergreifende Lernziele ab. Damit legte Fritz Oser den Grundstein für Forschung, die nicht nur auf fachübergreifende Merkmale der Oberflächen- und Tiefenstruktur fokussierte, sondern fachspezifisch Folgen von Lernschritten zu untersuchen suchte - z. B. in Politik (Henkenborg et al. 2007), Sport (Jeisy 2013) oder Technik (Grimm 2009). Dabei zeigte sich verschiedentlich, dass sich die Einhaltung der Schrittfolgen spezifischer Basismodelle im Unterricht positiv auf den Lernerfolg auswirkt (Brouër 2001; Draxler 2005). In der Folge entstand z.B. in der Physikdidaktik eine ganze Serie von Forschungsarbeiten, die Physikunterricht hinsichtlich der Strukturiertheit und Vollständigkeit von Handlungsschritten und ihrer Wirkung auf Lernen untersucht haben (z. B. Reyer 2003; Geller 2015; Stender et al. 2013). Fritz Oser leistete damit einen substantiellen Beitrag nicht nur zur Weiterentwicklung der Unterrichtsforschung, sondern insbesondere auch zur fachdidaktischen Unterrichtsforschung.

\subsection{Basismodelle des Unterrichts in Lehrkräfteaus- und Weiterbildung}

Mit den Basismodellen hat Fritz Oser auch wesentlich dazu beigesteuert, (angehenden) Lehrkräften eine Folie für die Unterrichtsplanung und -reflexion zur Verfügung zu stellen. In der Folge haben die Basismodelle Einzug in die Lehrkräfteausbildung gehalten (Krabbe und Fischer 2020). Zum Beispiel setzen Buschhüter et al. (2020) die Basismodelle im Rahmen der Vorbereitung von Studierenden auf die Planung und Reflexion von Unterricht im Praxissemester ein. Aber auch in der Fortbildung von Lehrkräften haben sich die die Basismodelle bewährt. Trendel et al. (2008) nutzen die im Physikunterricht am häufigsten auftretenden drei Basismodelle Konzeptbildung, Lernen durch Eigenerfahrung und Problemlösen als Schablone für die gemeinsame Unterrichtsreflexion mit Lehrkräften und setzen in der Folge individuelles Coaching ein, um die Lehrkräfte darin zu unterstützen, abhängig vom Lehrziel das entsprechende Basismodell umzusetzen. Die Ergebnisse der Begleituntersuchung belegen in der Folge einen basismodellkonformeren Unterricht (Wackermann et al. 2010). Zander (2016) entwickelt die Fortbildung weiter und weist auch eine Wirkung auf den Lernerfolg nach, insbesondere bei Schüler*innen mit schwacher bis mittlerer Leistung. Damit hat sich Fritz Osers Idee der Basismodelle nicht nur für die Forschung, sondern auch für die Unterrichtspraxis als ausgesprochen hilfreich erwiesen.

In der Summe hat Fritz Oser in beeindruckender Weise mit einer einzigen Studie einen nachhaltigen Beitrag zur theoretischen Weiterentwicklung des Begriffs der Unterrichtsqualität geleistet, für zwölf verschiedene Lernziele Modelle guten Unterrichts formuliert sowie empirisch fundiert und damit eine nachhaltige Grundlage für die Vermittlung konkreten Wissens zur Gestaltung guten Unterrichts geschaffen, die nicht nur in der Allgemeinen Didaktik, sondern auch in der Fachdidaktik große Akzeptanz erlangt hat. 


\section{Fritz Oser und die neue Welt - das pädagogische Potenzial von Fehlern und negativem Wissen (Hans Gruber)}

Das pädagogische und erziehungswissenschaftliche Interesse am Umgang mit Fehlern, das ein Markenzeichen von Fritz Oser war, steht in bemerkenswertem Zusammenhang mit seiner Leidenschaft für Kunst und Kultur, insbesondere für die Musik. Als gelernter Geiger und Orchesterdirigent war er mit den Bedingungen des Gelingens oder Misslingens künstlerisch-kreativer Erfahrungen genügend vertraut, um keine Sekunde daran zu zweifeln, dass die Arbeit in jenen Grenzbereichen des zuvor Erreichten es ist, was den an eigenen Lern- und Entwicklungsprozessen interessierten Menschen voranbringt, auch wenn Fehlschläge dabei unvermeidbar sind. Sowohl Fehler als auch Kunst und Kultur allgemein haben die explizite, aber selten unkomplizierte Thematisierung wertbezogenen Handelns, Urteilens und Lebens gemeinsam: Für Fritz Oser galt hier wie dort, hörbar und sichtbar in der Wissenschaft und in der Gesellschaft Stellung zu beziehen und zu begründen, weswegen bestimmten Dingen, Strukturen oder Prozessen Wert beigemessen wird, und es galt ihm pädagogisch, das Interesse für Werte zur wecken und nachhaltig zu vermitteln, wie beispielsweise im Modell der just communities exemplifiziert.

Bei Fritz Oser war die Entscheidung, nach Abschluss seiner Promotion (mit einer Dissertation zum Thema „Das Gewissen lernen. Probleme intentionaler Lernkonzepte im Bereich der moralischen Erziehung") das Wagnis eines Post-Doc-Aufenthalts in den USA einzugehen und eine Blindbewerbung an Lawrence Kohlberg zu schicken (stolz zeigte er den Antwortbrief, auf dem unter Harvard-Briefkopf handschriftlich nur ,I take you. Kohlberg“ geschrieben war), sinnbildlich für die Entschlossenheit, sich um - nicht nur, aber ausdrücklich auch - religiöse und ethische Werte und Entscheidungen ebenso wie um den Umgang mit notwendigerweise damit verbundenen Unzulänglichkeiten lebenslang kümmern zu müssen.

Es ist nur natürlich, dass sich diese wissenschaftliche Entschlossenheit im Verlauf einer langen akademischen Karriere in unterschiedlichen pädagogischen Handlungsfeldern in anspruchsvollen und im positiven Sinne risikobehafteten Forschungsprogrammen äußerte. Fritz Oser zeigte, dass die Frage der angemessenen Beimessung menschlicher Werte für schulische Szenarien ebenso relevant ist wie für berufsbildende oder berufliche Konstellationen oder für private und persönliche Beziehungen, wenn sich auch Form und Ausmaß der für ethische Belange zur Rechenschaft heranziehbaren Akteure gravierend unterscheiden. Die unterschiedlichen Verantwortlichkeiten, Hierarchiestufen, Machtverhältnisse oder Verletzbarkeiten konstituieren wertbezogene Asymmetrien, aus denen pädagogische Verpflichtungen unweigerlich resultieren. Dies gilt für Lehrkräfte im Verhältnis zu ihren Schülerinnen und Schülern ebenso wie für Vorgesetzte und deren Teammitglieder, für Orchesterdirigent*innen in der Probenarbeit mit dem Ensemble ebenso wie für Eltern und deren Kinder und wie für partnerschaftliche Beziehungen.

Als eine Gemeinsamkeit der doch theoretisch wie methodisch so unterschiedlichen Zugänge erwies sich das Lernen aus Fehlern, das Fritz Oser als wichtigen Teil menschlichen Lebens und Zusammenlebens auffasste, auch wenn er sich in seinen erziehungswissenschaftlichen Analysen und Forschungsarbeiten vor allem mit dem expertenhaften Handeln von Lehrkräften und Berufstätigen beschäftigte. Er hob die 
besondere Rolle von Fehlern für Lehr-Lern- und Erziehungsprozesse hervor, mahnte aber, dass hierfür Reflexion über die Rolle von Verantwortung (oder eben nicht) einzelner Menschen für von ihnen begangene Fehler, über die von Organisationen (und der Öffentlichkeit) zugeschriebene Verantwortung für Fehler und resultierende Konsequenzen (zum Beispiel im Umgang mit Untergebenen oder mit Schüler*innen), über die kognitive Repräsentation von Fehlern als Abweichungen normgerechten Handelns oder Denkens, über begünstigende Kontexte und Vorbedingungen für das Entstehen von Fehlern sowie über Möglichkeiten der Verarbeitung und (hoffentlich) künftigen Vermeidung von Fehlern erfolgen müsse. Solche Formen der Reflexion treten nicht notwendigerweise spontan auf, weswegen ihre Förderung eine wichtige pädagogische Aufgabe ist. Hierfür sah Fritz Oser in Fehlern besonderes Potenzial - auch wenn (oder gerade weil) Fehler negativ konnotiert sind, vermögen sie es, die Menschen zu ,bewegen“. Für Fritz Oser stellten sie daher sozusagen besonders günstige Konstellationen dar, in denen es gelingen könne, Menschen davon zu überzeugen, dass es an der Zeit sei, Verantwortung für den Umgang mit dem Erfahrenen zu übernehmen. Wenn dergestalt die eigenen Wissens-, Handlungs- und Urteilsstrukturen und -prozesse zum Gegenstand der Reflexion werden, steigt das Potenzial, über individuelle Verantwortungsübernahme neue Wege beschreiten zu können.

Damit erweist sich das als pädagogische Fundgrube, was auf den ersten Blick erschreckend wirkt: Alle Menschen machen Fehler, auch Lehrkräfte, Vorgesetzte, Trainer*innen, Eltern und Expert*innen. Fritz Oser wandte sich - in seiner Entschlossenheit und in der Explizitheit seiner theoretischen Aufarbeitung setzte er hier ebenso wie in der Entwicklung pädagogischer Modelle Meilensteine - entschieden gegen die Auffassung, Fehler nur als Ausdruck des Versagens anzusehen, also als Ereignisse, die mit Verfehlung und Scheitern verbunden sind. Wenn niemand gegen Fehler gefeit ist, kommt es umso mehr darauf an, wie sie oder er damit umgeht. Selbst dann, wenn trotz besten Bemühens Fehler geschehen, für die man die Verantwortung zu übernehmen hat, lässt sich darauf auf eine Art und Weise reagieren, die eine nachträglich positive Wendung einleitet - was den gezielten und reflexiven Umgang mit den Fehlern und die Bereitschaft zu deren schonungsloser Reflexion erfordert.

Im Rahmen einer Lehrveranstaltung erzählte Fritz Oser einmal eine Episode, die dies eindrücklich unterstreicht. Er war Mitglied der Prüfungskommission eines Referendars, der eine Lehrprobe mit einer Klasse der Mittelstufe im Biologieunterricht - Thema Fische - zu halten hatte. Dem bedauernswerten Referendar unterliefen offenbar Fehler beim classroom management, und so ereignete es sich, dass ein Schüler einen Fisch aus dem Aquarium holte und einer Mitschülerin ins T-Shirt steckte - mit allem vorstellbaren Aufruhr und Hallo im Klassenzimmer. Der Referendar vollzog einen „Unterbruch“ - er stoppte seinen Unterricht und thematisierte die moralische Tragweite und Relevanz des Vorfalls - seinen eigentlichen Lehrproben-Auftrag offenbar völlig vergessend. Fritz Oser berichtete, dass sich die Mitglieder der Prüfungskommission einig waren, dass das Unterrichtskonzept und damit die Lehrprobe vollkommen gescheitert waren, dass dies aber dennoch die beste Unterrichtsstunde gewesen sei, die sie gesehen hatten. 
In Fritz Osers Augen war es klar, dass das Erfolgsmodell, das sowohl dem Konzept der just community als auch dem Konzept negativen Wissens zugrunde liegt, ist, dass die Behandlung wert- und moralbezogener Fragen Vorrang vor inhaltlichen Fragen genießen muss und die Einordnung inhaltlicher Themen auf einem höheren reflexiven Niveau erlaubt. Fehler sind oft ein Anlass für solche Prozesse, und pädagogisch angemessen, so Fritz Osers Botschaft, ist es, solche Gelegenheiten nicht ungenutzt zu lassen.

Eine wichtige Voraussetzung für die pädagogische Nutzbarkeit von Fehlersituationen ist es natürlich, hierfür wissenschaftliche Voraussetzungen zu schaffen. Fritz Oser beklagte es, dass die meisten psychologischen, erziehungs- oder bildungswissenschaftlichen Konzepte an dem Umgang mit kanonisch strukturiertem, ,richtigen“ Wissen und an korrekten Prozessen ausgerichtet sind und damit keine geeignete bzw. hinreichende Grundlage für den Umgang mit Fehlern bieten. Daher plädierte er einerseits in der Erziehungswissenschaft eindringlich dafür, Fehler und den Umgang mit ihnen als pädagogisch reizvolles und wichtiges Thema zu adeln, stellte aber andererseits zugleich hierfür die theoretischen und analytischen Grundlagen bereit. Dies erfolgte besonders deutlich in seiner Konzeption einer ungewöhnlichen und auf den ersten Blick in sich widersprüchlichen Wissensform, dem ,negativen Wissen“. $\mathrm{Zu}$ den konstruktiven Strategien, die mit der Arbeit mit negativem Wissen verbunden sind, zählen die offene Ursachensuche, die kollektive Reflexion und die Entwicklung besserer Handlungsmuster - allesamt mutige Folgerungen aus Fehlern, denen doch allzu oft mit Schuldzuweisung an einzelne Personen begegnet wird. Aus Fehlern gelerntes Wissen ist häufig negatives Wissen darüber, was falsch ist, was man nicht tun sollte oder wo eigene Schwächen und Defizite liegen.

In dem Sammelband „Fehlerwelten: Vom Fehlermachen und Lernen aus Fehlern“ (Althof 1999) wurden empirische und konzeptuelle Zugänge (weiter)entwickelt, unter denen die Theorie des negativen Wissens (Oser et al. 1999) besondere Bedeutung besitzt und sich als besonders eindrücklich und nachhaltig erweist. In der Forschung zeigte sich, dass es eine enorme Varianz darin gibt, welche Bedeutung und Wertschätzung dem Umgang mit Fehlern und mit negativem Wissen in verschiedenen Praxisfeldern beigemessen wird. Die Schule ist häufig gekennzeichnet durch ein Wissens- und Machtgefälle der Akteure, das mit der epistemologischen Deutungshoheit einhergeht: die Lehrkräfte verfügen über das kanonisch gesicherte (,richtige") Wissen und Handlungskenntnisse, die die Schüler*innen erwerben sollen. Die spontane reflexive Erarbeitung des pädagogischen Potenzials von Fehlern und negativem Wissen fällt in solchen Konstellationen schwer. In beruflichen Konstellationen spielen organisationale Merkmale eine Schlüsselrolle dabei, ob Fehler zu gezielten Lernprozessen oder zu Vertuschungsversuchen führen. Letztere werden vor allem eingesetzt, wenn Fehler zu negativen beruflichen Konsequenzen (bis hin zur Entlassung) führen; sie finden sich oft in Berufsfeldern, deren Selbstverständnis auf Konzepten von Fehlerfreiheit und Stabilität aufbauen, etwa dem Bankensektor. Die gezielte Thematisierung, Hinterfragung und Analyse von Fehlern sowie der Versuch, das negative Wissen der Beschäftigten über Fehlerquellen, Fehlervariationen und Fehlerkonsequenzen zu erweitern, finden sich etwa in der Flugsicherung oder bei Pilot*innen, aber auch im professionellen Sport, in dem videogestützte Analysen beruflichen Handelns an der Tagesordnung sind. 
In seinen letzten Lebensjahren arbeitete Fritz Oser insbesondere daran, weiteres Licht in die Thematik zu bringen, wann, wo und wie ein Sachverhalt nachvollziehbar als fehlerhaft beurteilt werden kann und welches Kriterium oder welche Norm durch ihn verletzt wurde, um darauf basierend pädagogische Maßnahmen zu begründen (Oser 2015). Er entwickelt eine drei Ebenen umfassende Systematik der Fehlerkonsequenzen, nämlich (1) die Wahrnehmung von Fehlern oder der eigenen Fehlbarkeit, (2) lineare Konsequenzen von Fehlern, z. B. im Sinne von resultierenden Wissensbeständen, die sich unmittelbar aus der Auseinandersetzung mit Fehlern entwickeln, sowie (3) nicht-lineare Konsequenzen des Lernens aus Fehlern, also Brüche oder gar radikale Neuanfänge in einer Entwicklung. Insbesondere der letzte Aspekt lag ihm am Herzen, und es ist kein Wunder, dass er wieder die Musik bemühte, um die besondere Bedeutung der nicht linearen Reaktion auf Fehler und auf Scheitern zu verdeutlichen:

Einer der schönsten Chöre von Joseph Haydn in seiner Schöpfung bezieht sich auf den Sturz der Höllengeister Schar in des Abgrunds Tiefen begleitet von Verzweiflung, Wut und Schrecken, und anschließend die großartige Vertonung des „Und eine neue Welt entspringt auf Gottes Wort“. Bildlich gesehen würde dies - auch wenn wir wissen, dass Metaphern nie genau eine gelebte Welt treffen - den Prozess des Loslösens vom Falschen und die Befreiung aus ihm in ein Neues, ein Unbekanntes, das erst noch entstehen muss, bedeuten. (Oser 2015, S. 71)

Seine Analyse, inwiefern sich die bisherige erziehungswissenschaftliche Forschung zu Fehlern und zu negativem Wissen vor allem mit linearen Reaktionen beschäftigte, führte zu drei Befunden. Erstens wird negativem Wissen eine Schutzfunktion zugeschrieben, mit der erreicht werden soll, dass ein Fehler nicht wiederholt wird, dass also in gewohnte Fahrwasser zurückgefunden wird. Zweitens wird angenommen, dass richtiges Wissen und Verhalten gerade durch die Abgrenzung vom Falschen zusätzliche stabile Sicherheit erlangt. Schließlich wird drittens unterstellt, dass durch Kontrastbildung das Bestehende eine umso klarere Kontur gewinnt, an dem Orientierung möglich ist.

In dem angesprochenen Text führt Fritz Oser an wundervollen Beispielen wie dem Scheitern von anscheinend auf Dauer gebauten Freundschaften auf, dass diese linear auf das Richtige verweisenden Funktionen von Fehlern zwar nicht falsch, sondern oft sinnvoll sind, dass die zugrundeliegende wissenschaftliche Perspektive jedoch viel zu kurz greift, weil sie nicht berücksichtigt, dass Fehlern auch das Potenzial innewohnt, ,dass etwas völlig Neues entsteht, das das Alte zwar abstößt, aber einen substantiellen Mehrwert enthält" (Oser 2015, S. 71).

Die weitere Umsetzung dieser spannenden, nachgerade revolutionären Ideen, die den Weg in eine neue Welt eröffnen können, muss nun leider ohne Fritz Osers Drängen und Inspirieren gelingen. 


\section{Ohne Zauberformel und Anabolika: Moralerziehung als Forschungsschwerpunkt von Fritz Oser (Martin Rothgangel)}

Ungeachtet seines reichhaltigen und vielfältigen Schaffens gibt es kaum ein Thema im Lebenswerk von Fritz Oser, das er intensiver als die Moralerziehung bearbeitet hätte. Ohne seiner entsprechenden Publikationstätigkeit gerecht werden zu können, sollen folgende Veröffentlichungen als Beleg dienen: Osers Dissertation setzt sich mit dem Thema „Das Gewissen lernen“ (Oser 1976) auseinander, seine Habilitation aus dem Jahr 1979 trägt den Titel „Das moralische Urteil in Gruppen“ (Oser 1981); es folgen zahlreiche weitere Publikationen über diese Thematik (z.B. Berkowitz und Oser 1985; Oser et al. 1986a, 1986b; Oser und Althof 1992; Oser 1998; Garz et al. 1999; Edelstein et al. 2001; Oser und Spychiger 2005; Heinrichs et al. 2013; Drahmann und Oser 2019) bis hin zu seinem Todesjahr (Forster-Heinzer und Oser 2020).

\subsection{Innovationen und Impulse Fritz Osers für die Unterrichtsforschung}

Bestimmte Innovationen Fritz Osers mögen gegenwärtig als bildungswissenschaftlicher Standard erscheinen, waren es aber zum Zeitpunkt ihrer Publikation nicht: Hervorgehoben sei Osers Forschungsaufenthalt an der Harvard University bei Lawrence Kohlberg von 1976 bis 1977 und die daraus resultierenden Impulse. So besteht ein Verdienst Osers darin, dass er entscheidend ,zur Rezeption der Kohlberg'schen Moralpsychologie und -pädagogik im deutschen Sprachraum beigetragen" (Bucher 2007, S. 11) hat. Damit verbunden ist der Impuls, entwicklungs- und moralpsychologische Erkenntnisse für die Moralpädagogik zu nutzen sowie die Etablierung von ,just community-Elementen in Schulen der Schweiz und der Bundesrepublik“ (ebd., S. 11). Dabei geht es um eine Gestaltung von Schulen als eine demokratische Gemeinschaft, wodurch soziales Verstehen, Verantwortungsbereitschaft und demokratische Einstellungen gelebt und gelernt werden sollen.

Weitere wichtige Innovationen sind Osers Studien zum negativen moralischen Wissen, d.h. „Wissen darüber, was ungerecht, unwahrhaftig, korrupt, katastrophal nachlässig, trivial böse, irreversibel hässlich, unsozial, Vorteil ausnutzend, unsolidarisch, untreu, parteiisch etc. und dies auf der deklarativen wie der prozeduralen Ebene ist" (Oser 2005, S. 171). In diesen Arbeiten wird eine Forscherpersönlichkeit erkennbar, die auf der einen Seite begriffliche Klarheit anstrebt, die Funktionen des negativen moralischen Wissens herausarbeitet und auf empirische Studien rekurriert - aber auf der anderen Seite ebenso klar die Grenzen des gegenwärtigen Wissensstandes markiert und den Gedankengang mit einem Forschungsdesiderat beschließt: „die Frage, wie viel Unmoral ein Mensch braucht, um moralisch zu werden, oder, wie viel negatives moralisches Wissen aufgebaut werden muss, hat noch keine Antwort gefunden“"(Oser 2005, S. 180). Last but not least ist seine Forschung zum Berufsethos von Lehrpersonen anzuführen, wobei anstelle von Tugendkatalogen ein realistisches - insbesondere auch konfliktspezifisches - Diskursmodell tritt (Oser 1998). 


\subsection{Fritz Osers Einfluss auf gegenwärtige Forschung und Unterrichtspraxis}

Im Folgenden wird exemplarisch anhand von zwei Handbüchern zu „Moral and Character Education“ (Nucci et al. 2014) bzw. „ethischer Bildung“ (Lindner und Zimmermann 2021) der Frage nachgegangen, welche moralpsychologischen bzw. pädagogischen Aspekte von Fritz Osers Wirksamkeit gegenwärtig rezipiert werden. Im erstgenannten Handbuch findet sich zum einen der von Fritz Oser verfasste Artikel „Toward a Theory of the Just Community Approach“ (Oser 2014, S. 198-222). Zum anderen wird in diesem Handbuch eine breite Palette von Osers Forschungstätigkeit aufgegriffen: Als Weiterführung zu Kohlbergs Methode der Dilemma-Diskussion wird Osers gruppenzentrierte Methode der Diskursethik angeführt (S. 75; vgl. S. 165), seine Forschung zu just community schools (S. 78), zur Berufsmoralität von Lehrpersonen (S. 101) sowie zur negativen Moral (S. 131). Im deutschsprachigen Handbuch ethischer Bildung von Lindner und Zimmermann (2021) werden Publikationen von Fritz Oser hinsichtlich folgender Aspekte rezipiert: just community (S. 40), negatives moralisches Wissen (S. 263, 331), Kohlberg-Rezeption (S. 270, 311, 315) sowie Diskursdidaktik (S. 311). Darüber hinaus wird in diesem Handbuch an einer Stelle deutlich, dass Oser (hier in Verbindung mit Althof) durch seine anschauliche Art und Weise der Argumentation eine Fundgrube für Zitate ist: „Es gibt, so Fritz Oser in seinem Lehrbuch über moralische Selbstbestimmung, keine Zauberformel des moralischen Lernens, keine Tricks und keine Anabolika, durch die moralische Kraft entsteht“" (S. 378 mit Bezug auf Oser und Althof 1992, S. 11).

Resümierend ist diese Durchsicht beider Handbücher ein Hinweis darauf, dass gegenwärtig weitgehend diejenigen Aspekte von Osers moralpsychologischer und -pädagogischer Forschungstätigkeit rezipiert werden, die schon im ersten Abschnitt hervorgehoben und ganz ähnlich bereits von Bucher (2007, S. 11) genannt wurden (u. a. Kohlberg-Rezeption, just community).

\subsection{Fritz Osers potenzieller Einfluss auf die zukünftige Entwicklung}

Die obigen Ausführungen legen es nahe, dass auch zukünftig Fritz Osers weiterführende Rezeption von Kohlbergs Moralpsychologie und -pädagogik, seine differenzierten Analysen zum Berufsethos sowie zum negativen moralischen Wissen einen wichtigen Bezugspunkt wissenschaftlicher Forschung darstellen werden. Genauso bedeutsam erscheint mir jedoch ein weiterer Punkt, der bei Osers Forschungstätigkeit zur moralischen Erziehung gleichfalls deutlich hervortritt, aber weniger eine Innovation darstellt, sondern eher mit seinem Forschungsinteresse und -habitus verknüpft ist: Seine Forschungstätigkeit ist zwar durch eine konsequente empirische Forschung sowie durch den interdisziplinären Dialog zwischen Moralpsychologie und -pädagogik gekennzeichnet. Darüber hinaus treten jedoch zwei weitere Punkte hervor, die in der Regel nicht eigens gewürdigt werden: Erstens Fritz Osers philosophische Expertise, welche durch sein Studium der Philosophie grundgelegt ist. Diese tritt in seinen Publikationen zur Moralerziehung u. a. durch seine Auseinandersetzung mit Jürgen Habermas und John Rawls immer wieder hervor (z. B. Oser 1981, 1998) und ist etwa für eine angemessene Berücksichtigung der Sein-SollenDifferenz unabdingbar. 
Neben dieser philosophischen Expertise ist aber noch ein weiterer Aspekt für das Wirken Fritz Osers konstitutiv: Das Interesse am Praxisbezug. Schon früh lassen sich praxisbezogene Publikationen etwa zur Friedensbildung (Oser und Friemelt 1973) oder auch entsprechende Ausführungen im Kontext seiner Dissertation greifen (vgl. das Vorwort von Widmer in Oser 1976, S. 7). Aber auch später ist für ihn, dem international ausgewiesenen Experten bildungswissenschaftlicher Moralforschung, dieses konsequente Praxisinteresse eine stete Triebfeder seiner Forschungstätigkeit. Es bleibt zu wünschen, dass für die zukünftige Entwicklung der Unterrichtsforschung dieser von Fritz Oser so beispielhaft praktizierte Dialog zwischen empirischer Forschung und philosophischer Reflexion sowie zwischen theoretischer Erkundung und praktischer Ausrichtung gleichfalls wegweisend ist.

\section{Mit Evidenz Dornröschen wachküssen: Fritz Oser und die Lehrer*innenbildung (Manfred Prenzel)}

Vor nicht langer Zeit sah Fritz Oser die (Schweizer) Lehrer*innenbildung in einem Dornröschenschlaf und hoffte, ihr das Erwachen durch evidenzbasierte Neuerungen versüßen zu können (Oser et al. 2017). Es war ein ständiges Anliegen seines wissenschaftlichen Schaffens, theoretisch begründete Evidenz für die Weiterentwicklung der Lehrer*innenbildung zu gewinnen und zur Diskussion zu stellen. Dass viele für die Lehrer*innenbildung relevanten Beiträge von Fritz Oser mit seinen Arbeiten in weiteren Forschungsfeldern (wie Wertebildung oder Unterrichtschoreographien) verwoben sind, liegt auf der Hand. Aber auch seine Beiträge, die sich mit Fragen der Lehrer*innenbildung befasst haben, behandeln eine beeindruckende Vielfalt an Themen. Auf zwei Schwerpunkte dieser Arbeiten möchte ich im Folgenden näher eingehen.

\subsection{Starke Impulse}

Fritz Oser hat sich - so intensiv und beständig wie wohl niemand anderer - mit dem Berufsethos von Lehrer*innen befasst (Oser 1994, 1995, 1998, 2018; Oser et al. 1991). Dass jemand, der zu moralischer und religiöser Entwicklung geforscht hat, das Ethos von Lehrer*innen untersucht, überrascht nicht. Doch geht es Fritz Oser dabei nicht um bestimmte Tugenden, die Lehrer*innen auszeichnen und von anderen Menschen abheben. Es geht ihm auch nicht um eine besondere Qualität eines pädagogischen Bezugs. Im Gegenteil: Sein Verständnis von einer pädagogischen Beziehungsqualität kann man als Gegenpol zu reformpädagogischen Mystifizierungen dieses Bezugs sehen, die zudem besondere Gefühlsausprägungen auf der Seite der Lehrer*innen unterstellen - und damit ein professionelles Nähe-Distanz-Verhältnis zwischen Lehrer*innen und Schüler*innen fast ausschließen. Fritz Oser sieht die Beziehung zwischen Lehrer*innen und Schüler*innen sehr wohl geprägt durch Fürsorge und Kümmern, aber nicht durch ein einseitiges Helfen. Vielmehr ist die durch Verantwortung geprägte Beziehung darauf angelegt, dass die Schüler*innen selbst zunehmend Verantwortung übernehmen. Es sind Maximen wie Lernen erleichtern, Kommunikation ermöglichen, Sinn stiften, Gerechtigkeit walten lassen, 
die nach Fritz Oser das Berufsethos von Lehrer*innen bestimmen. Wiederum wird der Anspruch der Gerechtigkeit nicht einfach moralisch eingefordert, sondern als professionelle Verpflichtung begründet. Diese besteht darin, den Schüler*innen das Lern- und Unterstützungsangebot zukommen zulassen, das dem besten (pädagogisch-didaktischen, lernpsychologischen) Wissen entspricht: Entsprechend müssen sich Lehrer*innen professionell weiterentwickeln und diskursfähig sein. So finden sich bereits in früheren Schriften von Fritz Oser bemerkenswerte Argumentationen: Sie nehmen die Lehrer*innenbildung in die Pflicht, die Entwicklung eines umfassenden, auf professionellem Wissen beruhenden Berufsethos (insbesondere Verantwortlichkeit und Wirksamkeit) anzubahnen und zu unterstützen.

Vorstellungen von einem Berufsethos fließen auch in den zweiten Arbeitsschwerpunkt zur Lehrer*innenbildung ein, der hier als Beispiel für Fritz Osers Schaffen angesprochen wird. Bereits eine ganze Zeit bevor in Deutschland die Diskussion um Bildungsstandards begann, hat Fritz Oser (1997a, 1997b) Standards für Lehrer*innenbildung entworfen und untersucht. Diese Standards bestimmen und prägen, so Fritz Oser, eine Profession. Sein theoretisches Modell umfasst 88 Standards, die er zwölf Gruppen zuordnet und für die entsprechenden Kompetenzen von Lehrer*innen stehen. Die Standardgruppen decken ein sehr breites Spektrum von professionellen Anforderungen ab, die nicht nur den Unterricht, sondern auch die Schulentwicklung und die Selbstorganisation der Lehrkraft betreffen. Auch wenn Fritz Oser konzediert, dass sein Modell noch unvollständig und weiter zu erforschen ist, umfasst es bereits zentrale Aufgaben, wie zum Beispiel das Diagnostizieren, das Begleiten von Lernprozessen, den Aufbau von sozialem Verhalten, die Leistungsmessung und die „Medien“. Die Konzeption der Standards beruht auf Gesprächen mit Expert*innen. Inwieweit die Standards erfüllt sind, untersucht Fritz Oser mit raffiniert abgestuften Selbsteinschätzungen, die ebenfalls darauf hinweisen, welchen Beitrag die Lehrer*innenbildung zur Entwicklung dieser Kompetenzen geleistet hat. So wird der Ansatz systematisch genutzt, um die Wirksamkeit der Lehrer*innenbildung in der Schweiz zu untersuchen (Oser und Oelkers 2001) und empirische Evidenz zu gewinnen, die das schlummernde Dornröschen in Bewegung bringen könnte.

\subsection{Nachhaltige Inspirationen}

Nimmt man das Beispiel „Standards in der Lehrerbildung“, dann kann Fritz Oser sowohl in theoretischer wie empirischer Hinsicht als Vorreiter für die nachfolgende große Welle empirischer Studien zu Kompetenzen von Lehrer*innen gelten. Auch die „Standards für die Lehrerbildung“, die 2004 von der KMK verabschiedet werden, lassen Fritz Osers Ideen erkennen. Fritz Oser selbst hat das Thema weiterverfolgt und seinen Ansatz weiterentwickelt, speziell für die Untersuchung von Kompetenzprofilen von Lehrpersonen in der Beruflichen Bildung (Oser et al. 2013). Aber auch die vertiefenden Analysen zu TEDS-M und die Rückbezüge auf die Lehrer*innenbildung in der Schweiz (Oser et al. 2015) werden durch seine Vorstellungen von Standards angereichert.

Fritz Osers Arbeiten zum Berufsethos wurden in den Communities, die Kompetenzen von Lehrer*innen untersuchen, durchaus aufgegriffen und geschätzt. Doch scheint das Konzept des Berufsethos von Lehrer*innen für viele Kolleg*innen zu 
komplex und zu anspruchsvoll, vor allem empirisch überhaupt nicht leicht umzusetzen zu sein. Aber alle in der Community verweisen auf Fritz Oser, wenn das Berufsethos von Lehrer*innen thematisiert wird. So hat er viele Anstrengungen unternommen, um Kolleg*innen für Forschung zum Berufsethos zu begeistern. Und das ist gelungen; mit Spannung wird das internationale Handbuch zum Berufsethos von Lehrer*innen erwartet, das Fritz Oser (Oser et al. im Druck) noch mit aller Kraft vorangetrieben hat.

Fritz Osers Arbeiten haben viele Kolleg*innen inspiriert und stimuliert, kluge Forschungsfragen zu relevanten Aspekten der Lehrer*innenbildung weiterzuverfolgen. Seine theoretischen Ausführungen sprühen von Ideen, stellen sich der Komplexität, sind aber immer so angelegt, dass empirische Zugänge möglich werden. Egal, ob Fritz Oser alleine oder mit unterschiedlichen Teams forschte, trugen die empirischen Zugänge seine Handschrift. Sie waren einerseits pragmatisch an den zur Verfügung stehenden Ressourcen ausgerichtet, andererseits unkonventionell und originell, ja mutig (wenn man etwa an seine Studien zur Wirksamkeit der Lehrer*innenbildung denkt) und erlaubten zügiges Forschen. Dabei gelang es Fritz Oser insbesondere aufgrund seiner theoretischen Souveränität immer hoch anregende Erkenntnisse aus diesen Studien zu ziehen.

\subsection{Botschaften für morgen}

Fritz Oser hat mit seinen Studien begonnen, eine von Traditionen geprägte Lehrer*innenbildung theoretisch und empirisch kritisch zu durchleuchten. Als entscheidendes Kriterium für die Beurteilung der Wirksamkeit der Lehrer*innenbildung galt ihm die Befähigung von Lehrer*innen, ein umfassendes Spektrum von Bildungszielen zu verfolgen und Schule und Unterricht nach dem besten Wissen (und damit förderlich und gerecht) zu gestalten. Fritz Oser nutzte den Kompetenzbegriff, aber es ging ihm (vermutlich mehr als anderen Forscher*innen in diesem Feld) letztlich um die Umsetzung in Handeln in praktischen Situationen. Damit rückten akademische Wissensstrukturen für ihn eher in den Hintergrund. Wichtiger war ihm die Kohärenz der Lehrer*innenbildung, die er gefährdet sah, etwa aufgrund einer unzureichenden Abstimmung von Allgemeiner Didaktik, Fachdidaktik und Pädagogischer Psychologie und einer mangelnden Koordinierung der Lehrer*innenbildung über Phasen bzw. über die Berufsbiographie.

Zentrale Botschaften - auch aus jüngeren Schriften - von Fritz Oser sind aus meiner Sicht: Wir müssen sehr viel ernsthafter und umfassender die Wirkung und Wirksamkeit unserer Lehrer*innenbildung empirisch prüfen! Wir müssen der Frage nachgehen, wie Lehrer*innen Wissen aus unterschiedlichen Bereichen integrieren und - auf dieser Grundlage - in anspruchsvollen Situationen handlungsfähig und wirksam werden. Schließlich sollten wir uns im Sinn von Fritz Oser nicht scheuen, auch große, empirisch nicht so leicht zu bearbeitende Fragen anzugehen. Wir müssten uns zum Beispiel viel intensiver mit dem Berufsethos von Lehrer*innen befassen, in der Forschung wie im Studium und in der Praxis!

Funding Open Access funding enabled and organized by Projekt DEAL. 
Open Access Dieser Artikel wird unter der Creative Commons Namensnennung 4.0 International Lizenz veröffentlicht, welche die Nutzung, Vervielfältigung, Bearbeitung, Verbreitung und Wiedergabe in jeglichem Medium und Format erlaubt, sofern Sie den/die ursprünglichen Autor(en) und die Quelle ordnungsgemäß nennen, einen Link zur Creative Commons Lizenz beifügen und angeben, ob Änderungen vorgenommen wurden.

Die in diesem Artikel enthaltenen Bilder und sonstiges Drittmaterial unterliegen ebenfalls der genannten Creative Commons Lizenz, sofern sich aus der Abbildungslegende nichts anderes ergibt. Sofern das betreffende Material nicht unter der genannten Creative Commons Lizenz steht und die betreffende Handlung nicht nach gesetzlichen Vorschriften erlaubt ist, ist für die oben aufgeführten Weiterverwendungen des Materials die Einwilligung des jeweiligen Rechteinhabers einzuholen.

Weitere Details zur Lizenz entnehmen Sie bitte der Lizenzinformation auf http://creativecommons.org/ licenses/by/4.0/deed.de.

\section{Literatur}

Althof, W. (Hrsg.). (1999). Fehlerwelten: Vom Fehlermachen und Lernen aus Fehlern. Opladen: Leske + Budrich.

Berkowitz, M.W., \& Oser, F. (1985). Moral education. Theory and application. Hillsdale: Erlbaum.

Brouër, B. (2001). Förderung der Wahrnehmung von Lernprozessen durch die Anwendung der Basismodelle des Lernens bei der Gestaltung von Unterricht. Unterrichtswissenschaft, 29(2), 153-170.

Bucher, A. (2007). Einführung und Vorschau auf den Band. In A. Bucher (Hrsg.), Moral, Religion, Politik. Psychologisch-pädagogische Zugänge. Festschrift für Fritz Oser (S. 9-17). Wien/Berlin: LIT.

Buschhüter, D., Mutschler, T., Schröder, J., Riese, J., \& Borowski, A. (2020). Basismodelle in Unterrichtsplanungen im Praxissemester Physik. In S. Habig (Hrsg.), Naturwissenschaftliche Kompetenzen in der Gesellschaft von morgen. Gesellschaft für Didaktik der Chemie und Physik Jahrestagung in Wien 2019 (S. 447-450). Essen: Universität Duisburg Essen.

Drahmann, M., \& Oser, F. (2019). Ethos, Moral und Werte - eine begriffliche und sachliche Klärung unterschiedlicher Konzepte. In C. Cramer \& F. Oser (Hrsg.), Ethos: interdisziplinäre Perspektiven auf den Lehrerinnen- und Lehrerberuf (S. 37-55). Münster/New York: Waxmann.

Draxler, D. (2005). Aufgabendesign und basismodellorientierter Physikunterricht. Dissertation, Universität Duisburg-Essen.

Drollinger-Vetter, B. (2011). Verstehenselemente und strukturelle Klarheit. Münster: Waxmann.

Edelstein, W., Oser, F., \& Schuster, P. (2001). Moralische Erziehung in der Schule.Entwicklungspsychologie und pädagogische Praxis. Weinheim u. a.: Beltz.

Forster-Heinzer, S., \& Oser, F. (2020). Berufsethos als Gegenstand der Lehrerinnen- und Lehrerbildung. In C. Cramer, J. König, M. Rothland \& M. Blömeke (Hrsg.), Handbuch Lehrerinnen- und Lehrerbildung (S. 105-113). Bad Heilbrunn: Julius Klinkhardt.

Frey, K., Frey-Eiling, A., Frei, A., \& Landolt-Marazzi, E. (1988). Allgemeine Didaktik. Zürich: Verlag der Fachvereine.

Garz, D., Oser, F., \& Althof, W. (Hrsg.). (1999). Moralisches Urteil und Handeln. Frankfurt/M.: Suhrkamp. Gebauer, H. (2016). Kognitive Aktivierung im Musikunterricht. Münster: LIT.

Geller, C. (2015). Lernprozessorientierte Sequenzierung des Physikunterrichts im Zusammenhang mit Fachwissenserwerb: Eine Videostudie in Finnland, Deutschland und der Schweiz. Berlin: Logos.

Grimm, A. (2009). Lehrerhandeln im gewerblich-technischen Unterricht - Ergebnisse einer empirischen Untersuchung zum Lehrerhandeln von Studienreferendarinnen und Studienreferendaren der beruflichen Fachrichtungen Elektrotechnik/Metalltechnik. Lernen \& Lehren, 93, 32-36.

Heinrichs, K., Oser, F., \& Lovat, T. (2013). Handbook of moral motivation. Theories, models, applications. Rotterdam/Boston/Taipei: Sense.

Henkenborg, P., Krieger, A., Pinseler, J., \& Behrens, R. (2007). Demokratie lernen und leben durch kognitive Anerkennung. Eigenverantwortliches Arbeiten und Lernen im Unterricht. Eine praxisorientierte Zusammenfassung. https://marvin.sn.schule.de/ sud/pdfs/tu_dresden.pdf. Zugegriffen: 26. Nov. 2020.

Jeisy, E. (2013). Choreografien des Lernens und Lehrens im Fachbereich Bewegung und Sport. Eine design-based-research-Studie zur Entwicklung von Lern-Lehrmodellen für die Lernverlaufsgestaltung im Sportunterricht. Dissertation, Universität Salzburg. 
Krabbe, H., \& Fischer, H. E. (2020). Gestaltung von Unterricht. In E. Kircher, R. Girdwidz \& H. E. Fischer (Hrsg.), Physikdidaktik (S. 117-153). Heidelberg: Springer.

Nucci, L., Narvaez D. \& Krettenauer T. (2014) (Hrsg.), Handbook of Moral and Character Education, 2. Aufl. New York/London: Routledge

Lindner, K., \& Zimmermann, M. (Hrsg.). (2021). Handbuch ethische Bildung. Stuttgart: UTB.

Oser, F. (1976). Das Gewissen lernen. Probleme intentionaler Lernkonzepte im Bereich der moralischen Erziehung. Olten: Walter. Mit einem Vorwort von Konrad Widmer.

Oser, F. (1981). Moralisches Urteil in Gruppen, Soziales Handeln, Verteilungsgerechtigkeit. Stufen der interaktiven Entwicklung und ihre erzieherische Stimulation. Frankfurt/M.: Suhrkamp.

Oser, F. (1994). Moral perspectives on teaching. Review of Research in Education, 20, 57-127.

Oser, F. (1995). Wann lernen Lehrer ihr Berufsethos? In A. Leschinsky (Hrsg.), Die Institutionalisierung von Lehren und Lernen. Beiträge zu einer Theorie der Schule. Festgabe für Peter Martin Roeder (S. 235-243). Weinheim: Beltz.

Oser, F. (1997a). Standards in der Lehrerbildung. Teil 1: Berufliche Kompetenzen, die hohen Qualitätsmerkmalen entsprechen. Beiträge zur Lehrerinnen- und Lehrerbildung, 15(1), 26-37.

Oser, F. (1997b). Standards in der Lehrerbildung. Teil 2: Wie werden Standards in der schweizerischen Lehrerbildung erworben? Erste empirische Ergebnisse. Beiträge zur Lehrerinnen- und Lehrerbildung, 15(2), 210-228.

Oser, F. (1998). Ethos - die Vermenschlichung des Erfolgs. Zur Psychologie der Berufsmoral von Lehrpersonen. Opladen: Leske + Budrich.

Oser, F. (2005). Negatives Wissen und Moral. In D. Benner (Hrsg.), Erziehung - Bildung - Negativität (S. 171-181). Weinheim: Beltz.

Oser, F. (2014). Toward a theory of the just community approach: effects of collective moral, civic, and social education. In L. Nucci, D. Narvaez \& T. Krettenauer (Hrsg.), Handbook of moral and character education (2. Aufl. S. 198-222). New York/London: Routledge.

Oser, F. (2015). „Und eine neue Welt ...“Funktionen des Negativen Wissens. Oder: Wenn Fehler Früchte tragen. In M. Gartmeier, H. Gruber, T. Hascher \& H. Heid (Hrsg.), Fehler: Ihre Funktionen im Kontext individueller und gesellschaftlicher Entwicklung (S. 71-92). Münster: Waxmann.

Oser, F. (2018). Unterrichten ohne Ethos? In H. Schärer \& M. Zutavern (Hrsg.), Das Professionelle Ethos von Lehrerinnen und Lehrern (S. 57-72). Münster: Waxmann.

Oser, F., \& Althof, W. (1992). Moralische Selbstbestimmung. Modelle der Entwicklung und Erziehung im Wertebereich. Ein Lehrbuch. Stuttgart: Klett-Cotta.

Oser, F., \& Baeriswyl, F. J. (2001). Choreographies of teaching: bridging instruction to learning. In V. Richardson (Hrsg.), Handbook of research on teaching (S. 1031-1065). Washington, DC: American Educational Research Association.

Oser, F., \& Friemelt, A. (1973). Den Frieden lernen. Christentum und Wissenschaft auf der Suche nach Frieden. Olten: Walter.

Oser, F., \& Oelkers, J. (Hrsg.). (2001). Die Wirksamkeit der Lehrerbildungssysteme. Von der Allrounderbildung zur Ausbildung professioneller Standards. Chur: Rüegger.

Oser, F., \& Patry, J.-L. (1990). Choreographien unterrichtlichen Lernens. Basismodelle des Unterrichts. Fribourg: Universität Fribourg.

Oser, F., \& Patry, J.-L. (1994). Sichtstruktur und Basismodelle des Unterrichts: Über den Zusammenhang von Lehren und Lernen unter dem Gesichtspunkt psychologischer Lernverläufe. In R. Olechowski \& B. Rollett (Hrsg.), Theorie und Praxis: Aspekte empirisch-pädagogischer Forschung (S. 138-146). Frankfurt: Peter Lang.

Oser, F., \& Spychiger, M. (2005). Lernen ist schmerzhaft. Zur Theorie des negativen Wissens und zur Praxis der Fehlerkultur. Weinheim u. a.: Beltz.

Oser, F., Althof, W., \& Garz, D. (Hrsg.). (1986a). Moralische Zugänge zum Menschen - Zugänge zum moralischen Menschen. München: Kindt.

Oser, F., Bauder, T., Salzmann, P., \& Heinzer, S. (Hrsg.). (2013). Ohne Kompetenz keine Qualität. Entwickeln und Einschätzen von Kompetenzprofilen bei Lehrpersonen und Berufsbildungsverantwortlichen. Bad Heilbrunn: Klinkhardt.

Oser, F., Biedermann, H., Brühwiler, C., \& Steinmann, S. (Hrsg.). (2015). Zum Start bereit? Vertiefende Ergebnisse aus TEDS-M zur schweizerischen Lehrerbildung im internationalen Vergleich. Opladen: Budrich.

Oser, F., Biedermann, H., \& Brühwiler, C. (2017). Die schweizerische Lehrerbildung im Dornröschenschlaf. Journal für LehrerInnenbildung, 17(3), 17-22.

Oser, F., Fatke, R., \& Höffe, O. (Hrsg.). (1986b). Transformation und Entwicklung. Grundlagen der Moralerziehung. Frankfurt/M.: Suhrkamp. 
Oser, F., Hascher, T., \& Spychiger, M. (1999). Lernen aus Fehlern. Zur Psychologie des negativen Wissens. In W. Althof (Hrsg.), Fehlerwelten: Vom Fehlermachen und Lernen aus Fehlern (S. 11-41). Opladen: Leske + Budrich.

Oser, F., Patry, J.-L., Zutavern, M., Reichenbach, R., Klaghofer, R., Althof, W., \& Rotbucher, H. (1991). Der Prozess der Verantwortung. Berufsethische Entscheidungen von Lehrerinnen. Schlussbericht. Freiburg: Pädagogisches Institut der Universität Freiburg.

Oser, F., Heinrichs, K., Bauer, J., \& Lovat, T. (Hrsg.). (in Druck). The international handbook of teacher ethos: strengthening teachers, supporting learners. Dordrecht: Springer.

Praetorius, A.-K., Herrmann, C., Gerlach, E., et al. (2020). Unterrichtsqualität in den Fachdidaktiken im deutschsprachigen Raum - zwischen Generik und Fachspezifik. Unterrichtswissenschaft, 48, 409-446.

Prenzel, M., Seidel, T., Lehrke, M., Rimmele, R., Duit, R., Euler, M., \& Widodo, A. (2002). Lehr-Lernprozesse im Physikunterricht - eine Videostudie. Zeitschrift für Pädagogik, 45, 139-156. Beiheft.

Reyer, T. (2003). Oberflächenmerkmale und Tiefenstrukturen im Unterricht: exemplarische Analysen im Physikunterricht der gymnasialen Sekundarstufe. Berlin: Logos.

Stender, A., Geller, C., Neumann, K., \& Fischer, H.E. (2013). Der Einfluss der Unterrichtstaktung auf die Strukturiertheit und Abgeschlossenheit von Lernprozessen. Zeitschrift für Didaktik der Naturwissenschaften, 19, 189-202.

Trendel, G., Wackermann, R., \& Fischer, H.E. (2008). Lernprozessorientierte Fortbildung von Physiklehrern. Zeitschrift für Pädagogik, 54(3), 322-340.

Wackermann, R., Trendel, G., \& Fischer, H.E. (2010). Evaluation of a theory of instructional sequences for physics instruction. International Journal of Science Education, 32(7), 963-985.

Zander, S. (2016). Lehrerfortbildung zu Basismodellen und Zusammenhänge zum Fachwissen. Berlin: Logos. 